OPEN ACCESS

Edited by:

Giulio Fracasso,

University of Verona, Italy

Reviewed by:

Alessandro Rizzo,

National Cancer Institute Foundation

(IRCCS), Italy

Genshu Wang,

Sun Yat-sen University, China

${ }^{*}$ Correspondence:

Baoxian Liu

liubxian@mail.sysu.edu.cn

Yunpeng Hua

hyp0427@163.com

${ }^{\dagger}$ These authors have contributed equally to this work and share first authorship

Specialty section: This article was submitted to Cancer Immunity and Immunotherapy, a section of the journal

Frontiers in Immunology

Received: 15 November 2021 Accepted: 03 January 2022

Published: 31 January 2022

Citation:

Shen J, Shen H, Ke L, Chen J, Dang $X$, Liu $B$ and Hua $Y$

(2022) Knowledge Mapping of Immunotherapy for Hepatocellular Carcinoma: A Bibliometric Study.

Front. Immunol. 13:815575. doi: 10.3389/fimmu.2022.815575

\section{Knowledge Mapping of Immunotherapy for Hepatocellular Carcinoma: A Bibliometric Study}

\author{
Jianming Shen ${ }^{1 \dagger}$, Hui Shen ${ }^{2 \dagger}$, Lixin $\mathrm{Ke}^{1 \dagger}$, Jialin Chen ${ }^{1}, \mathrm{Xi} \mathrm{Dang}^{1}$, Baoxian $\mathrm{Liu}^{2 *}$ \\ and Yunpeng $\mathrm{Hua}^{1 *}$ \\ ${ }^{1}$ Hepatobiliary and Pancreatic Center, the First Affiliated Hospital, Sun Yat-sen University, Guangzhou, China, ${ }^{2}$ Department \\ of Medical Ultrasonics, the First Affiliated Hospital, Sun Yat-sen University, Guangzhou, China
}

Background: Hepatocellular carcinoma $(\mathrm{HCC})$ is one of the most common malignant tumors, and many patients are diagnosed with advanced disease. The treatment of advanced liver cancer has made significant strides in recent years, owing to the practice of immunotherapy drugs. Numerous studies have been published on immunotherapy for HCC; however, no relevant bibliometric study has been published. This study aims to gain a better understanding of the current situation and to identify potential new research directions by conducting a bibliometric analysis on immunotherapy for HCC.

Methods: We searched the Web of Science Core Collection (WoSCC) for articles related to immunotherapy for HCC. Three software (VOSviewer, CiteSpace, and python) were primarily used to assess the contribution and co-occurrence relationships of various countries/regions, institutes, journals, and, authors as well as to identify research hotspots and promising future trends in this research field.

Results: A total of 1,641 English articles published between 2011 and 2020 were collected, with the number of articles increasing nearly every year. The majority of publications originated from China ( $n=893,54.42 \%$ ), followed by the United States and Japan. The Sun Yat-sen University contributed the most publications ( $n=97,5.91 \%)$. Nakatsura Tetsuya $(n=26)$ and Llovet JM $(n=366)$ were ranked first in the top ten authors and co-cited authors. Cancer Immunology Immunotherapy was the most productive academic journal on immunotherapy for HCC [ $n=46,2.80 \%$; impact factor (IF) $2020=6.9679]$. Aggregation and identification of critical nodes in the co-cited network demonstrated a shift in the field of HCC immunotherapy. Initially, the hotspots were predominantly "glypican-3", "cytokine-induced killer cells", and "ny-eso-1", while the emphasis has shifted in recent years to "landscape", "camrelizumab", "combination therapy", and "immune score".

Conclusion: Increased attention has been paid to HCC with the advancement of immunotherapy. At the moment, the most active frontiers are focused on better understanding the immunological landscape of liver cancer, screening the population that can benefit from immunotherapy, and the clinical application of immune checkpoint inhibitors, particularly in combination with other therapeutic options (such as local therapy and targeted therapy).

Keywords: HCC, Immunotherapy, Bibliometric study, VOSviewer, Citespace 


\section{INTRODUCTION}

Hepatocellular carcinoma (HCC) is the sixth most commonly diagnosed cancer and the fourth leading cause of cancer-related deaths, worldwide (1). Additionally, the majority of HCC patients are already at an intermediate-advanced stage when their diagnosis is confirmed, necessitating palliative care (2).

Tumor immunotherapy, as a novel and successful therapeutic strategy, has a broad prospect in advanced HCC. While there are diverse immunotherapeutic approaches available for treating HCC, including immune checkpoint inhibitors, peptide vaccines, dendritic cell vaccines, chimeric antigen receptor $\mathrm{T}$ cells, and oncolytic viruses (3), immunotherapy trials utilizing immune checkpoint inhibitors have emerged as a major focus of research for cancer treatment (4). Immune checkpoint inhibitors (ICIs) are monoclonal antibodies directed primarily against several immune checkpoint proteins, including cytotoxic T-lymphocyte antigen 4 (CTLA-4), programmed cell death protein 1 (PD-1), and its ligand (PD-L1) in the tumor microenvironment (TME). In terms of liver cancer treatment, $\mathrm{PD}-1$ antibodies significantly improved the prognosis of patients with advanced liver cancer, achieving an objective response rate (ORR) of $17 \%-20 \%$ and a complete remission in some patients $(5,6)$. Given that ICI monotherapy appears to be effective only in a small subset of patients, the search for predictors of response to ICIs and combination therapy in patients with unresectable HCC has steadily gained interest (7). Additionally, targeted drugs in combination with ICIs have a much higher ORR in advanced liver cancer, indicating a favorable therapeutic potential, and the FDA has also approved it as a firstline treatment for unresectable or metastatic liver cancer (8-10). Therefore, immunotherapy may represent a significant novel concept and a promising future perspective in the study of HCC and become an indispensable part of the treatment regimen for HCC in the near future (11).

Over the last few decades, an increasing number of studies have been published on immunotherapy for HCC. There is, however, no literature to assess the published related literature systematically. Bibliometrics can use mathematical and statistical methods to quantitatively analyze a large number of documents in a particular research field, revealing numerous facets and research trends in that field $(12,13)$. At present, scientometric analysis of the literature is primarily performed using CiteSpace (14), VOSviewer (15), and HistCite (16). Numerous researchers have used this strategy to assess their respective research domains (17-20).

Nevertheless, no specific scientometric research on the knowledge mapping of HCC immunotherapy has been conducted to date. The present study evaluates the literature on immunotherapy for HCC from 2011 to 2020 to describe the current state of the field and identify new research directions.

\section{MATERIALS AND METHODS}

\section{Data Collection}

On September 27, 2021, we searched for relevant literature between 2011 and 2020 in the field of immunotherapy for
HCC using the Web of Science Core Collection (WoSCC). The Science Citation Index-Expanded (SCI-E) was used as the data source with the publication types were limited to "article". The main search terms were "primary liver carcinoma", "primary liver cancer", "immunotherapy", and "immunotherapeutic". The detailed search strategy is presented in the Supplementary Material. Two authors (HS and JS) independently searched the WoSCC database for relevant literature and downloaded the relevant information (title, keyword, author information, abstract, reference, etc.) in TXT format. Subsequently, the two authors (LK and JC) excluded articles that did not adhere to the aforementioned criteria. Divergent viewpoints would be resolved through discussions or a third party (BL and $\mathrm{YH}$ ).

\section{Statistical Methods}

Microsoft Office Excel 2019 (Microsoft, Redmond, Washington, USA) was used to process the data and construct a polynomial regression model $\left(\mathrm{f}(\mathrm{x})=\mathrm{p} 0 \mathrm{x}^{\mathrm{n}}+\mathrm{p} 1 \mathrm{x}^{\mathrm{n}-1}+\mathrm{p} 2 \mathrm{x}^{\mathrm{n}-2}+\mathrm{p} 3 \mathrm{x}^{\mathrm{n}-3}+\ldots+\mathrm{pn}\right)$ to predict the number of articles published in 2021. Python (Python Software Foundation, Wilmington, DE) was used to draw visual maps that intuitively depict the national distribution of publications. VOSviewer (1.6.11) was used to explore collaboration networks between authors/institutes/countries/ journals. In VOSviewer, nodes were used to represent countries, institutions, journals, and authors, and their size was determined by their co-occurrence frequency in titles and abstracts (21). CiteSpace 5.7.R5 (Chaomei Chen, Drexel University, USA) can extract keywords and references from publications with high citation bursts and construct a dual-map overlay for journals. Therefore, CiteSpace can be used to investigate the research trends in a certain topic (22). CiteSpace parameters included were as follows: link retaining factor $(\mathrm{LRF}=3)$, e for top $\mathrm{N}(\mathrm{e}=2)$, time span (2011-2020), years per slice (1), look back years (LBY = 8), links (strength: cosine, scope: within slices), selection criteria (g-index: $\mathrm{k}=25)$, and minimum duration $(\mathrm{MD}=1)$.

\section{RESULTS}

\section{Annual Growth Trend of Publications}

A total of 1,641 articles about immunotherapy for HCC that were published between 2011 and 2020 were obtained after searching the WoSCC database. As shown in Figure 1, the annual output has been increasing steadily since 2015 , reaching a peak in 2020 ( $n=406,24.74 \%)$. The year 2012 had the lowest number of articles published $(n=71,4.33 \%)$, and the annual average number of articles published was 164 . By fitting the data, we observed a statistically significant relationship between the year and the number of publications $\left(R^{2}=0.9717\right)$. According to the fitting curve, we estimated that the number of publications about immunotherapy for HCC would reach 470 in 2021.

\section{Countries/Regions and Institutions Analysis}

A total of 1,641 articles were from 58 countries. According to the publishing number, as shown in the bar graph (Figure 2A) and 


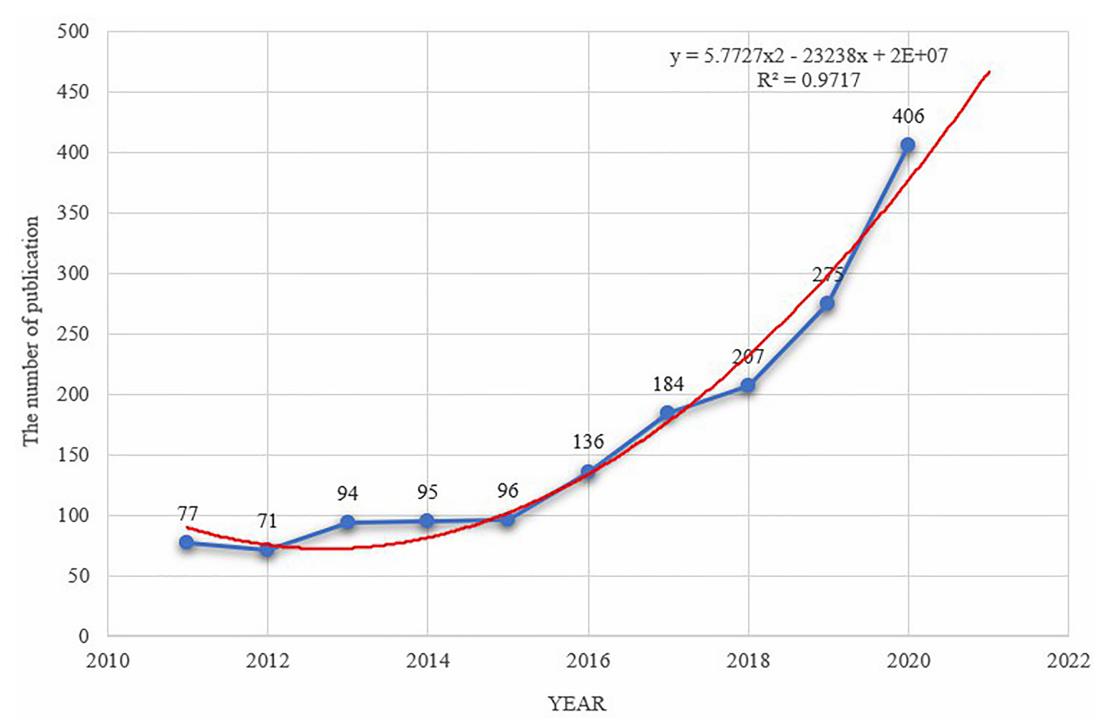

FIGURE 1 | The polynomial curve fitting of publication growth in immunotherapy of HCC.

the country distribution map (Figure 2B), the top 3 countries/ regions were China $(n=893,54.42 \%)$, United States $(n=392$, $23.89 \%)$, and Japan $(n=157,9.57 \%)$, respectively. Moreover, extensive cooperation between many countries/regions was also observed in Figure 2C, most notably between China and the United States.

These articles were contributed by 1,815 institutions, with the top 10 institutions contributing a total of 520 articles, accounting for $28.65 \%$ of all the articles (Figure 3A). Additionally, China was represented by seven of the top 10 institutions. Sun Yat-sen University ranked first $(n=97,5.91 \%)$, followed by Fudan University $(n=69,4.21 \%)$. As shown in Figure 3B, a collaboration between agencies was more extensive than that between countries. Sun Yat-sen University collaborated closely with many Chinese universities and research centers, and also with institutions from Singapore, the United States, and other countries.

\section{Authors and Co-Cited Authors}

More than 10,000 researchers were involved in HCC immunotherapy-related research. Among these, the top three authors with the most publications were Nakatsura Tetsuya $(n=$ 26), Kaneko Shuichi $(n=20)$, and Fan Jia $(n=19)$ (Table 1), respectively. Among the top 10 co-citation authors (Table 1), Llovet JM $(n=366)$ ranked first, followed by El-Khoueiry AB $(n=269)$, and Bruix $\mathrm{J}(n=260)$. VOSviewer was used to investigate co-authorship and citation networks between authors (Figure 4). Each node on the graph represents each author, the size of the circle reflects the number of articles published by the researcher, and the lines connecting the circles represent co-occurrence relationships between the authors. There was a close co-occurrence relationship between authors and co-cited authors, with more prolific authors often co-occurring more with other authors (Figure 4).

\section{Journals and Co-Cited Academic Journals}

There were a total of 466 academic journals publishing immunotherapy-related articles for liver cancer, with Cancer Immunology Immunotherapy ( $n=46$, IF $2020=6.9679)$ ranking first, followed by Oncoimmunology ( $n=46$, IF $2020=8.1097$ ). Among the top ten journals (Table 2), 40\% (4/10) were from the United States, followed by $20 \%(2 / 10)$ from the Netherlands and the United Kingdom. Simultaneously, among journals with more than 30 articles, the Journal of Hepatology $(n=31$, IF $2020=$ 25.0843) had the highest impact factor, followed by Hepatology $(n=31$, IF $2020=17.4243)$. As shown in Figure 5, there were positive citation relationships between different journals.

The dual-map overlay of journals in Figure $\mathbf{6}$ demonstrated the topic distribution of the journals. The citing journals were located on the left of the map, while the cited journals were located on the right. The labels represented the disciplines covered by the journals. From left to right, the colored lines depicted the citation paths. There were three distinct citation paths. Two orange citation paths suggested that studies from Molecular/Biology/Genetics journals and Health/Nursing/ Medicine journals were frequently cited in studies from the Molecular/Biological/Immunological journals. A green path suggested that studies from the Molecular/Biological/Genetic journals were frequently cited from studies in Medical/ Medicine/Clinical journals.

\section{Analysis of Co-Cited References}

Table 3 summarizes the top ten co-cited references. Among them, the paper entitled "Nivolumab in patients with advanced hepatocellular carcinoma (CheckMate 040): an open-label, noncomparative, phase 1 non-comparative 2 dose-escalation and expansion trial" published by El-Khoueiry AB et al. had the most co-citations $(n=256)$. Additionally, the literature with a 

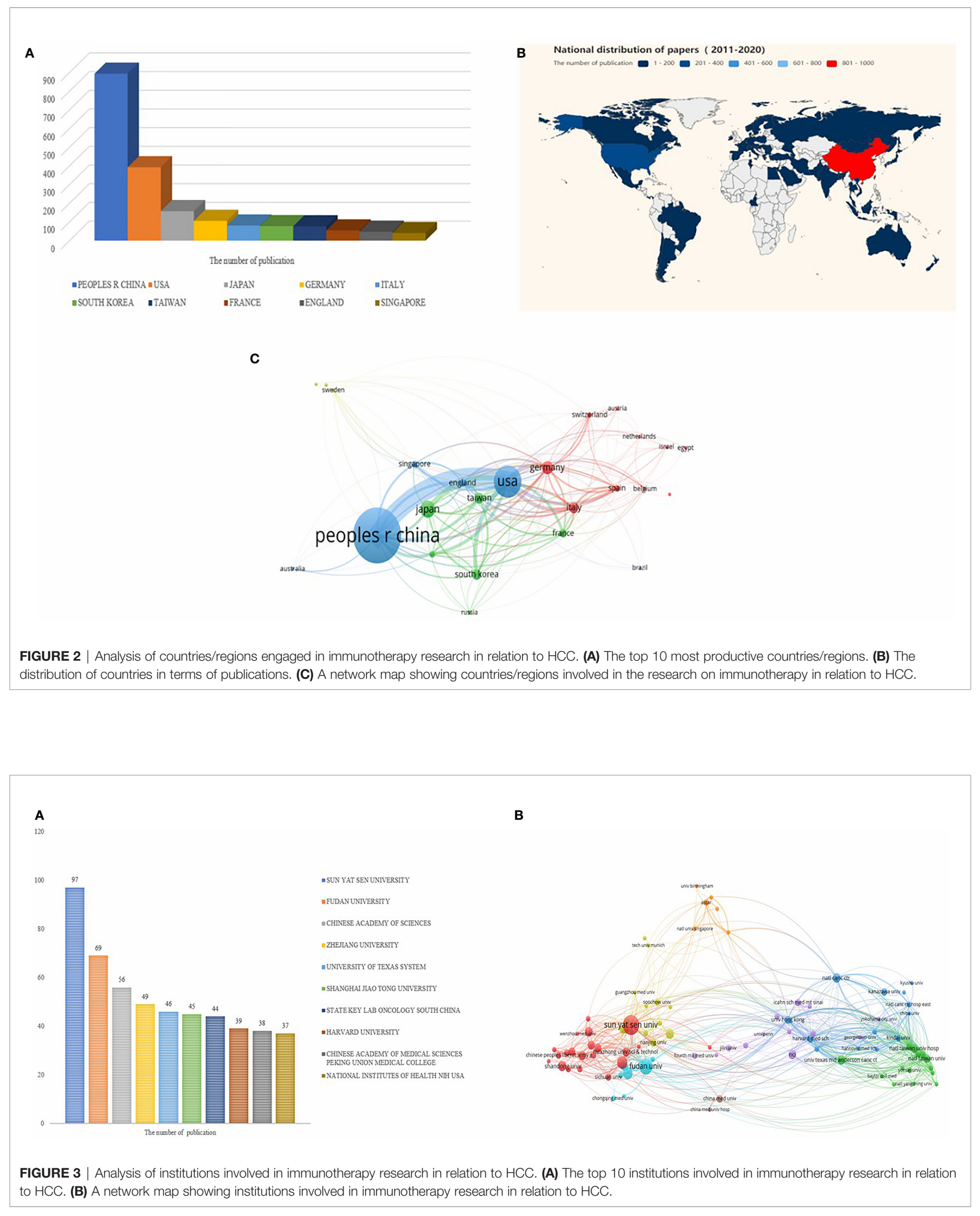
TABLE 1 | The top 10 authors and co-cited authors involved in research on immunotherapy in relation to HCC.

\begin{tabular}{|c|c|c|c|c|}
\hline Rank & Author & Count & Co-cited Author & Count \\
\hline 1 & Nakatsura Tetsuya & 26 & Llovet JM & 366 \\
\hline 2 & Kaneko Shuichi & 20 & EL-Khoueiry AB & 269 \\
\hline 3 & Fan Jia & 19 & Bruix J & 260 \\
\hline 4 & Mizukoshi Eishiro & 19 & Zhu AX & 181 \\
\hline 5 & Kudo Masatoshi & 18 & Kudo M & 171 \\
\hline 6 & Yoshikawa Toshiaki & 18 & Gao Q & 158 \\
\hline 7 & Zhou Jian & 17 & El-Serag HB & 158 \\
\hline 8 & Zheng Limin & 16 & Topalian SL & 158 \\
\hline 9 & Arai Kuniaki & 15 & Cheng AL & 128 \\
\hline 10 & Yamashita Tatsuya & 15 & Greten TF & 127 \\
\hline
\end{tabular}

total co-citation number of more than $100(n=4)$ was entirely composed of articles published in Lancet or one of its sub-journals.

CiteSpace was used to construct the network of co-cited references, and cluster analysis revealed 14 clusters (Figure 7A). Modularity Q (0.6764) and Mean Silhouette (0.761) values were both greater than 0.5 . The first cluster label on the knowledge map was "\#0 glypican-3" and the second cluster label was “\#1 programmed cell death-1". Simultaneously, we constructed a timeline display of co-cited references (Figure 7B). The timeline view is a methodology for visualizing data that combine clustering and time slicing techniques. Cluster labels are sorted according to whether they appear early or late after clustered, which not only illustrates the distribution of topics in this field but also displays the trend and interrelationship of study topics over time. In the Timeline view, different colors of nodes on the same line indicate different years. Therefore, the nodes on the left represent older references, while the nodes on the right represent more recent references. A straight line in the same horizontal position indicates the set of all clustered references belonging, and the cluster label is located at the line's rightmost end. The closest clusters on the timeline were "\#2 pd-11", “\#3 regorafenib", “\#4 immune score”, “\#6 murine model”, “\#7 combination therapy", "\#8 liver neoplasms", "\#10 prognostic", and "\#11 camrelizumab". CiteSpace was used to assess the references with a high citation burst. Citation bursts indicated that a reference had been widely cited over time and that the study findings of the references were well known in this field (Figure 8).
We found that among the top 15 references with the strongest citation bursts, "Zhu AX, 2018, LANCET ONCOL, V19, P940, DOI 10.1016/S1470-2045(18)30351-6" (2019-2020, strength 19.29) and "El-Khoueiry AB, 2017, LANCET, V389, P2492, DOI 10.1016/S0140-6736(17)31046-2” (2019-2020, strength 17.04) were the recent emergence of high-citation references.

\section{Analysis of Keywords}

The VOSviewer was employed to construct a network map of keywords (Figure 9A), and keywords with strong citation explosion were determined through CiteSpace (Figure 9B). In Figure 9B, the green line represents the time period from 2011 to 2020, while the periods of each burst keyword are plotted by the red line. The keywords that had citation bursts after 2015 were "vaccination" (2015-2017, strength 5.1), "resistance" (20182020, strength 6), "landscape" (2019-2020, strength 8.92), and "pd 1 blockade" (2019-2020, strength 5.66).

\section{DISCUSSION}

\section{General Information}

In the present study, we performed a systematic literature search of the Web of Science databases for articles published in the last decade about immunotherapy for HCC (2011-2020). After excluding studies that did not meet the screening criteria, this scientometric study comprised 1,641 English papers published in
A

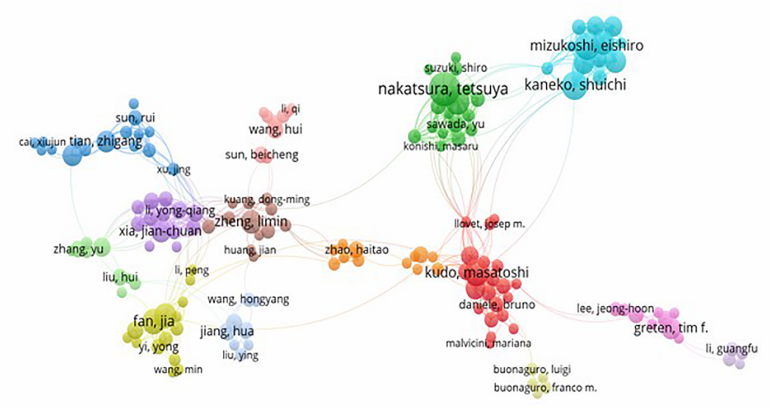

B

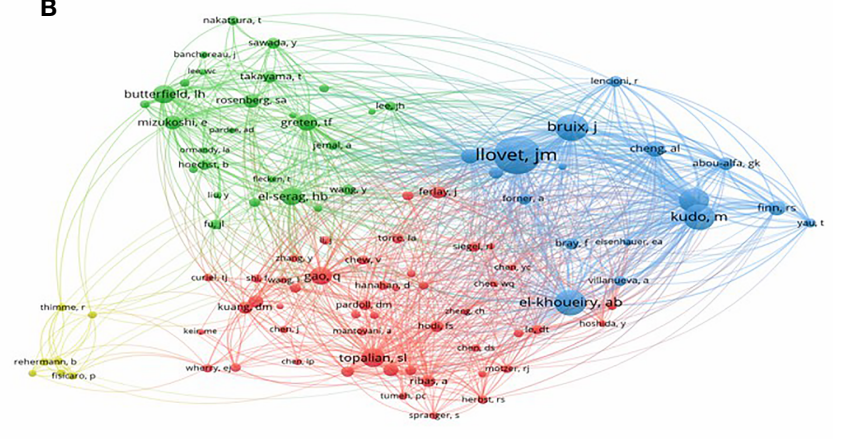

FIGURE 4 | A network map showing authors (A) or co-cited authors (B) involved in immunotherapy research in relation to HCC. 
TABLE 2 | The top 10 academic journals involved in immunotherapy research in relation to HCC.

\begin{tabular}{|c|c|c|c|c|c|}
\hline Rank & Journal & Count & Percent & Country & IF (2020)\# \\
\hline 1 & Cancer Immunology Immunotherapy & 46 & $2.80 \%$ & Germany & 6.968 \\
\hline 2 & Oncoimmunology & 46 & $2.80 \%$ & United States & 8.110 \\
\hline 3 & Oncotarget & 42 & $2.56 \%$ & United States & NA \\
\hline 4 & PLOS ONE & 39 & $2.34 \%$ & United States & 3.240 \\
\hline 5 & Journal for Immunotherapy of Cancer & 35 & $2.13 \%$ & United Kingdom & 13.752 \\
\hline 6 & Hepatology & 31 & $1.89 \%$ & United States & 17.424 \\
\hline 7 & Journal of Hepatology & 31 & $1.89 \%$ & Netherlands & 25.084 \\
\hline 8 & Oncology Letters & 25 & $1.52 \%$ & Greece & 2.968 \\
\hline 9 & International Immunopharmacology & 24 & $1.46 \%$ & Netherlands & 4.932 \\
\hline 10 & Scientific Reports & 22 & $1.34 \%$ & United Kingdom & 4.379 \\
\hline
\end{tabular}

\#IF, impact factor.

466 journals with 77,338 co-cited references from 1,815 institutions in 58 countries/regions.

As can be observed from the result, there is a general upward trend in the number of HCC immunotherapy-related publications, indicating that this topic has received significant attention in recent years. Additionally, three key inflection points occurred in the years 2015, 2018, and 2019. This phenomenon may be connected to significant events in the field of HCC immunotherapy. The US Food and Drug Administration (FDA) approved PD-1 checkpoint inhibitors (nivolumab and pembrolizumab) for use in non-small cell lung cancer and melanoma at the end of 2014, ushering in a new era of immunotherapy research for HCC. On September 30, 2017, the FDA authorized nivolumab for treatment in patients with advanced HCC (5). A year later, cancer immunotherapy using checkpoint inhibitors was awarded the Nobel prize. Following this, research on HCC immunotherapy-related research has advanced to a new level, and its clinical application has been a research hotspot in recent years.

While China was the only developing country to rank in the top 10 most productive countries/regions, it accounted for more than half of all publications. The explanation for this could be that China accounted for 55\% of all liver cancer cases globally, indicating that China is a country with a high incidence of liver cancer $(23,24)$. Institutional distribution was generally consistent with the country distribution. China contributed seven of the top 10 institutions, followed by the USA with three institutions. Close collaborations were observed between

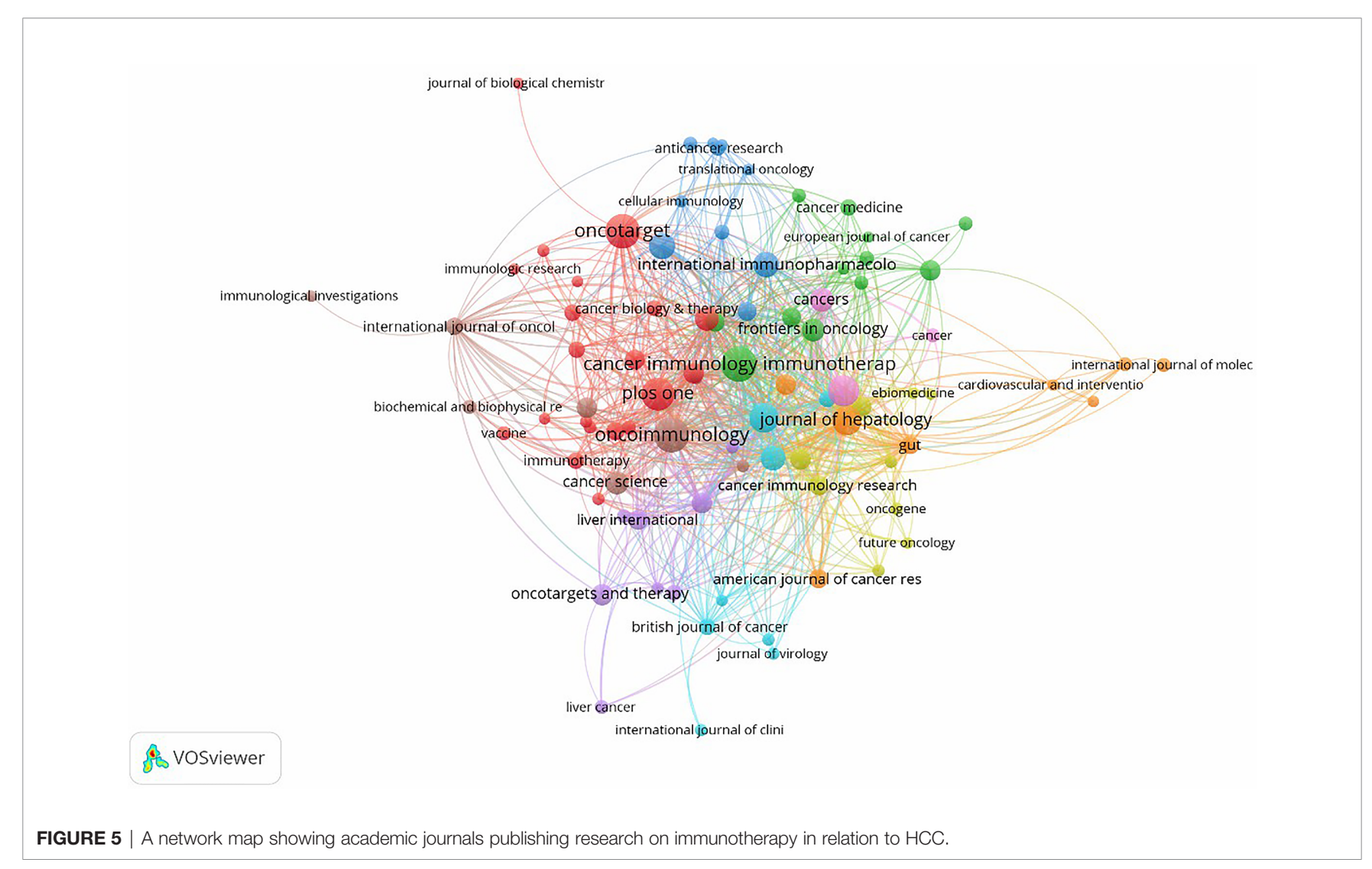




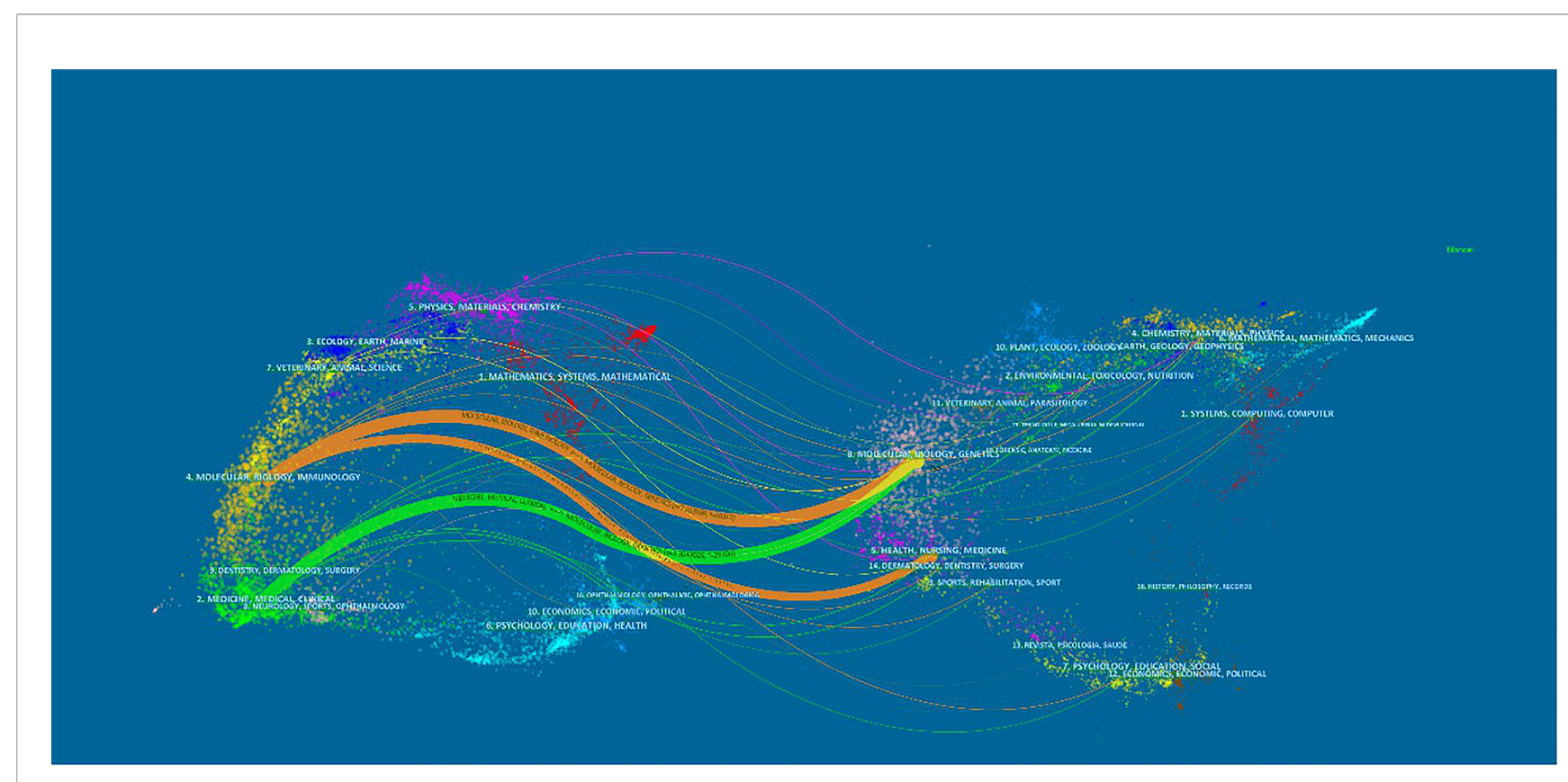

FIGURE 6 | A dual-map overlay of journals related to research on immunotherapy in HCC.

countries/regions and institutions; however, collaborations between agencies were found to be stronger than those between countries, implying that international collaborations should be strengthened (Figure 3B).

At least 15 articles were published by each of the top ten active authors. All of the top 10 active authors were from Asia, including 7 Japanese and 3 Chinese scholars. The finding may imply that Asian researchers play a significant role in HCC immunotherapy research and have made significant contributions. When co-cited authors are taken into account, the top 10 authors with at least 127 co-citations made significant contributions to the field of HCC immunotherapy. Llovet JM (366 co-citations) ranked first, followed by Anthony B El-Khoueiry (269 co-citations) and Bruix J (260 co-citations). Llovet JM is well known for his significant contributions to the formulation of liver cancer guidelines (25), the exploration of liver cancer treatment methods (26), and immunotherapy mechanisms (27). Anthony B El-Khoueiry has chaired several clinical trials evaluating immunotherapy drugs for the treatment of advanced liver cancer. CheckMate-040 (5) played a critical role in the FDA's approval of Opdivo (nivolumab) for the treatment of primary liver cancer patients who have failed to respond to sorafenib or have developed intolerance. Bruix J focused his research on targeted drugs for the treatment of liver cancer and contributed to the development of HCC management guidelines $(28,29)$, which were important in influencing the direction of HCC immunotherapy research.

Only 341 publications were published in the top ten academic journals, accounting for only $20.78 \%$ of all articles. Cancer Immunology Immunotherapy (30) ranked first in terms of total publications, followed by Oncoimmunology (30) and Oncotarget (31), indicating that these journals were particularly interested in articles regarding HCC immunotherapy research. These data will aid future scientists in selecting journals when submitting HCC immunotherapy-related manuscripts.

TABLE 3 | The top 10 co-cited references involved in research on immunotherapy in HCC.

\begin{tabular}{|c|c|c|c|c|}
\hline Rank & Co-cited reference & Count & Type & IF (2020)\# \\
\hline $\mathrm{R}-1$ & El-Khoueiry AB, 2017, Lancet, V389, P2492 & 256 & Clinical Trial & 79.321 \\
\hline $\mathrm{R}-2$ & Bruix J, 2017, Lancet, V389, P56 & 117 & Clinical Trial & 79.312 \\
\hline $\mathrm{R}-3$ & Zhu AX, 2018, Lancet Oncol, V19, P940 & 108 & Clinical Trial & 41.316 \\
\hline $\mathrm{R}-4$ & Kudo M, 2018, Lancet, V391, P1163 & 102 & Clinical Trial & 79.312 \\
\hline$R-5$ & Topalian SL, 2012, New Engl J Med, V366, P2443 & 94 & Clinical Trial & 91.245 \\
\hline $\mathrm{R}-6$ & Bray F, 2018, CA-Cancer J Clin, V68, P394 & 88 & Epidemiological Study & 508.702 \\
\hline $\mathrm{R}-7$ & Sangro B, 2013, J Hepatol, V59, P81 & 86 & Clinical Trial & 25.083 \\
\hline $\mathrm{R}-8$ & Pardoll DM, 2012, Nat Rev Cancer, V12, P252 & 75 & Review & 60.716 \\
\hline $\mathrm{R}-9$ & Prieto J, 2015, Nat Rev Gastro Hepat, V12, P681 & 74 & Review & 46.802 \\
\hline $\mathrm{R}-10$ & Ferlay J, 2015, Int J Cancer, V136, P0 & 73 & Epidemiological Study & 7.396 \\
\hline
\end{tabular}

\#IF, impact factor. 
A

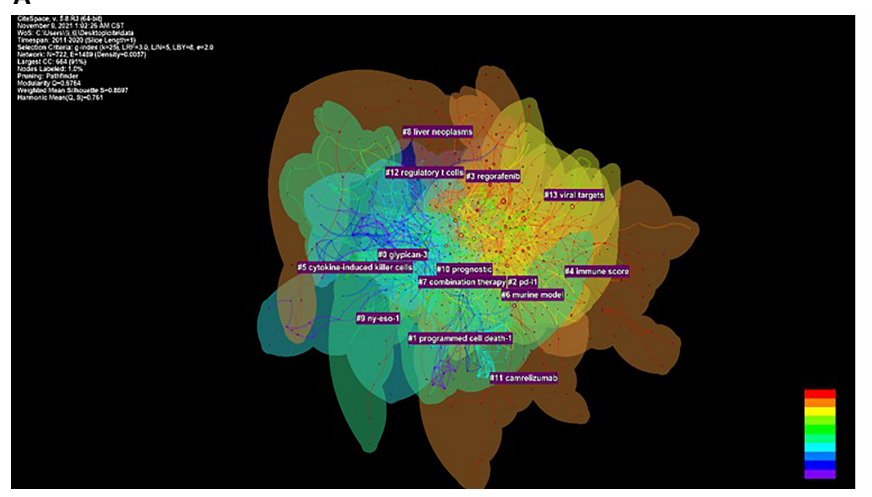

B

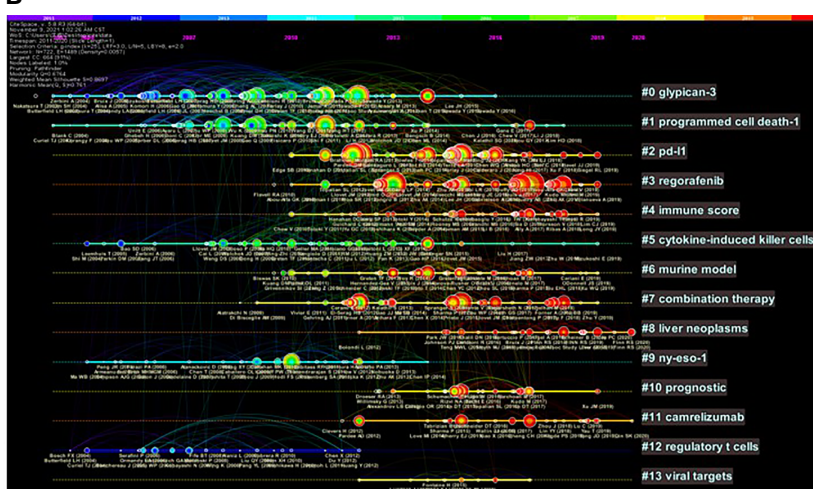

FIGURE 7 | The knowledge map (A) and the timeline view (B) of references related to research on immunotherapy in HCC.

Furthermore, after comprehensively analyzing the data of authors, co-cited authors, and co-cited references, we found that Masatoshi Kudo was the only scholar who appeared in all three indicators, suggesting that he is an accomplished author in this field, and his teams would make excellent potential collaborators for researchers.

\section{Knowledge Base}

When two publications are cited jointly by a third citing publication, this is referred to as a co-citation relationship (32). The more often a piece of literature is cited, the more significant it is perceived in a certain field. Therefore, the most frequently cited publications or high-impact literature can be viewed as a knowledge base and primary focus for researchers in a particular field. As shown in Table 3, they are mainly from top-ranked journals and composed of six clinical trials, two reviews, and two epidemiological studies.

Firstly, these six clinical studies provide an overview of the discovery and development process for HCC immunotherapy. Sorafenib ushered in the era of targeted therapy for liver cancer in 2007. Over the last 10 years, the exploration of targeted therapy for liver cancer was mostly stagnant, although immunotherapy showed promise. Topalian SL et al. (33) (R-5) conducted a multicenter phase I clinical trial (NCT00729664) in 2012 to evaluate the efficacy of anti-PD-L1 monoclonal antibodies in a variety of advanced solid tumors. Although

\section{Top 15 References with the Strongest Citation Bursts}

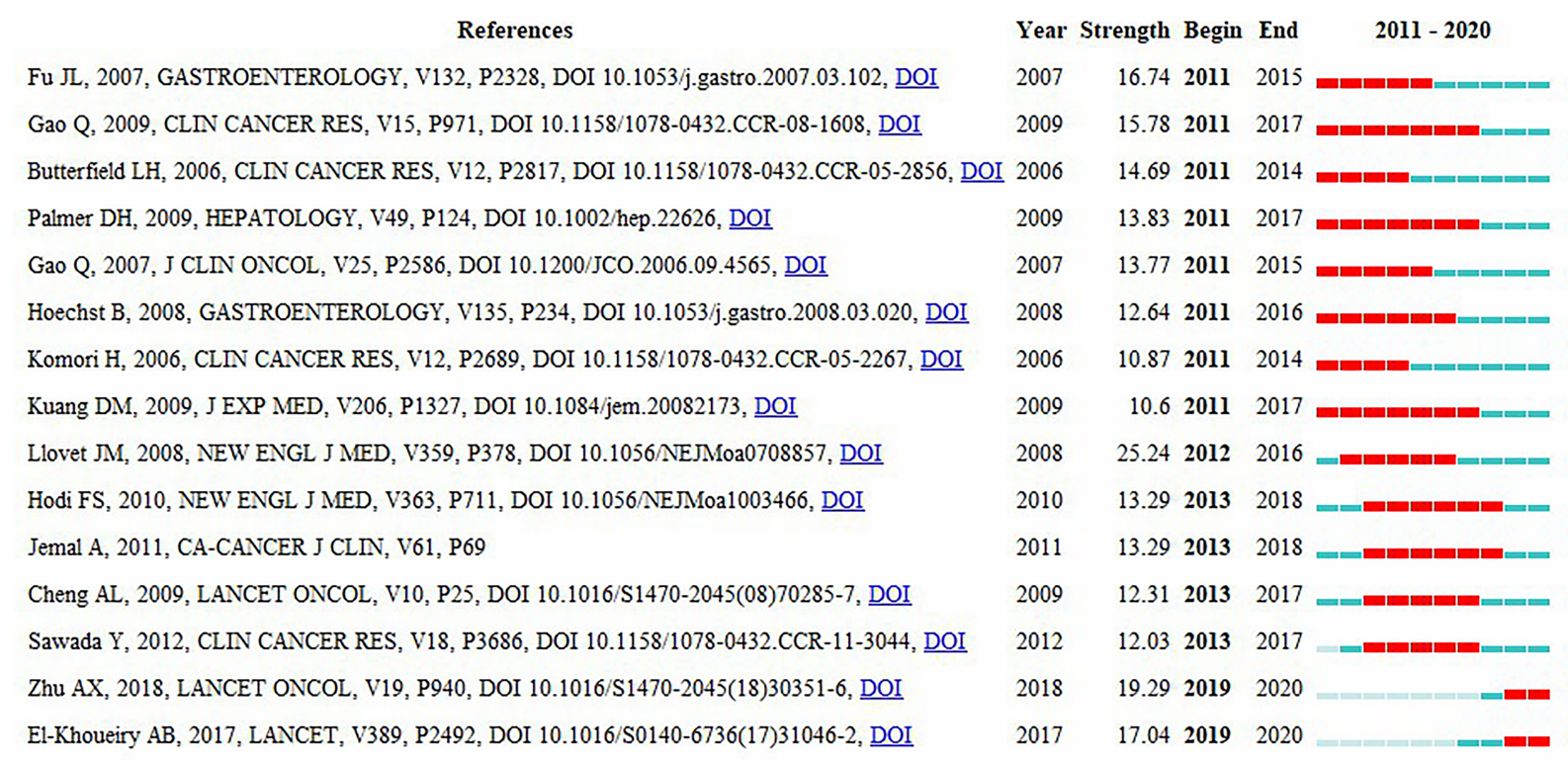

FIGURE 8 | Top 15 references with strongest citation bursts. 


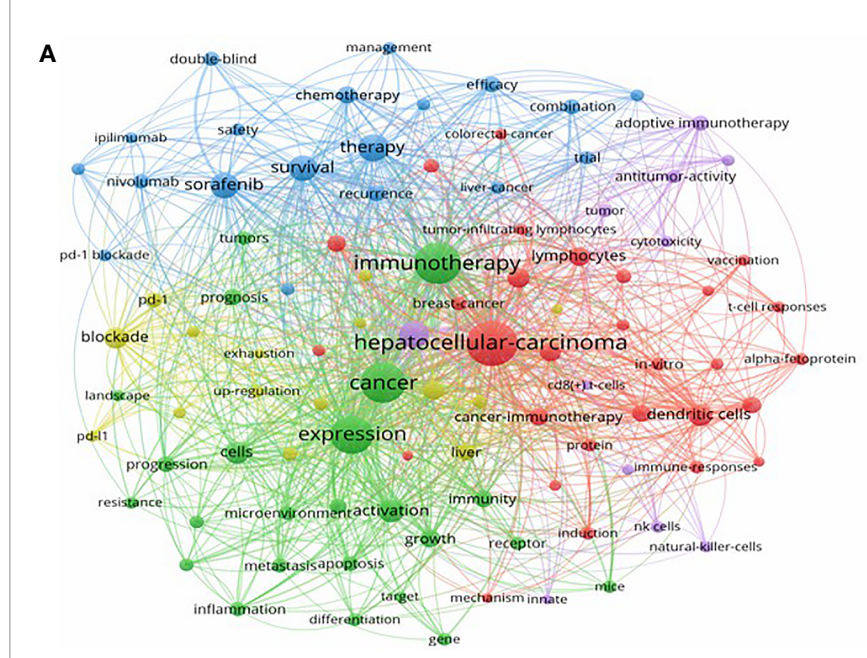

B Top 15 Keywords with the Strongest Citation Bursts

\begin{tabular}{|c|c|c|c|c|c|}
\hline Keywords & Year & Strength & Begin & End & $2011-2020$ \\
\hline dendritic cell & 2011 & 10.02 & 2011 & 2013 & $=$ \\
\hline adoptive immunotherapy & 2011 & 9.2 & 2011 & 2016 & =" \\
\hline$t$ cell response & 2011 & 7.73 & 2011 & 2015 & =-10 \\
\hline alpha fetoprotein & 2011 & 7.28 & 2011 & 2016 & 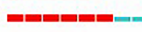 \\
\hline in vivo & 2011 & 6.08 & 2011 & 2014 & $=$ \\
\hline in vitro & 2011 & 5.46 & 2011 & 2016 & =ستص \\
\hline cytotoxic $\mathrm{t}$ lymphocyte & 2011 & 5.45 & 2011 & 2015 & = \\
\hline cancer patient & 2011 & 4.69 & 2011 & 2013 & $=$ \\
\hline phase ii & 2011 & 5.63 & 2012 & 2016 & - \\
\hline mice & 2011 & 4.63 & 2012 & 2017 & - \\
\hline phase i & 2011 & 4.55 & 2012 & 2013 & $-=$ \\
\hline vaccination & 2011 & 5.1 & 2015 & 2017 & - - \\
\hline resistance & 2011 & 6 & 2018 & 2020 & - \\
\hline landscape & 2011 & 8.92 & 2019 & 2020 & - \\
\hline pd 1 blockade & 2011 & 5.66 & 2019 & 2020 & \\
\hline
\end{tabular}

FIGURE 9 | (A) A network map of keywords. (B) The keywords with the strong citation bursts in articles related to immunotherapy research in HCC.

these patients enrolled in this study did not include liver cancer patients, the results demonstrated the safety and activity of antiPD-L1 antibodies in patients with advanced cancer, prompting the scholars to investigate the use of anti-PD-L1 in the treatment of advanced liver cancer. One year later, Sango et al. (34) (R-7) published the results of a clinical trial using CTLA- 4 antibody (tremelimumab) to treat HCC. The clinical trial showed that the CTLA-4 antibody exhibited antitumor and antiviral activity, with manageable adverse drug reactions. Over the next few years, pertinent clinical research was conducted. El-Khoueiry AB et al. (5) (R-1) published the most co-cited study (CheckMate040) in 2017, with 268 co-citations. This study reported the use of an anti-PD-1 antibody (nivolumab) to treat advanced HCC. The results showed that, regardless of whether or not they had received sorafenib treatment, nivolumab had a favorable response rate and overall survival time, and the drug was safe and tolerable. Simultaneously, another clinical trial with a novel targeted drug, regorafenib, achieved favorable findings the same year. Bruix J et al. (35) (R-2) reported this study (NCT01774344). The FDA approved regorafenib in April 2017 for patients with hepatocellular carcinoma who have previously received sorafenib treatment. Another clinical trial (Keynote-224) (NCT02702414) (R-3) published in 2018 by Zhu AX et al. (6) evaluated the safety and effectiveness of anti-PD-1 drug Pembrolizumab in 104 patients with advanced HCC who had previously received sorafenib. Similarly, Kudo M et al. (36) (R-4) presented the results of the REFLECT trial (NCT01761266), which compared overall survival in patients treated with lenvatinib against sorafenib as a first-line treatment for unresectable hepatocellular carcinoma. The FDA approved lenvatinib as a single-agent firstline treatment for patients with unresectable liver cancer based on the results of the REFLECT trial.

GLOBOCAN is a significant project of the International Agency for Research on Cancer (IARC), which tracks the cancer incidence, mortality, and cancer development trends of 36 types of cancer in 185 countries/regions worldwide, to summarize the global epidemiology of cancer and its impact on human health. In 2012 and 2018, Ferlay J et al. (37) (R-10) and Bray F et al. (24) (R-6) reported on cancer incidence and mortality worldwide, respectively. These two articles contributed significant epidemiological data on hepatocellular cancer, as evidenced by the fact that they were cited in several publications. In 2012 and 2015, Pardoll DM et al. (38) (R-8) and Prieto J et al. (39) (R-9) published two high-quality reviews on liver cancer immunotherapy, respectively. Both of them provided a complete overview of the immunotherapy mechanism for liver cancer at that time.

In general, these ten highly co-cited references showed the current epidemiology of liver cancer, the exploration, and development of immunotherapy for advanced liver cancer, as well as the clinical application of these treatments. Co-citation analysis can provide us with a wealth of useful information, allowing us to gain a better understanding of the evolution of the knowledge structure relating to HCC immunotherapy.

\section{Research Hotspots}

To further investigate and describe new immunotherapy hotspots for HCC, we used CiteSpace to examine the co-cited reference. As shown in Figure 7, early research focused on the investigation of various immunotherapy methods for HCC, such as "\#0 glypican-3", “\#5 cytokine-induced killer cells", and "\#9 nyeso-1", whereas current studies focused on “\#3 regorafenib”, “\#7 combination therapy", and "\#11 camrelizumab". Simultaneously, the present stage focuses on how to screen patients who react to immunotherapy, e.g., the "\#4 immune score".

Targeted therapy is a critical component of treatment for patients with advanced liver cancer, and regorafenib is one of the representative drugs (40). Although sorafenib has been on the market for more than a decade, the overall therapeutic result is insufficient, with approximately $30 \%$ of patients benefiting and these people typically developing drug resistance within 6 months (41). Regorafenib was approved by the FDA in 2017 as 
the second-line standard treatment for patients with advanced HCC after it was demonstrated to be efficacious in HCC patients with sorafenib resistance (35). Subsequent clinical studies on regorafenib demonstrated that it is a good and relatively safe treatment option for patients with advanced hepatocellular carcinoma (sorafenib resistance) (42). This may explain why regorafenib has become a study priority for academics over the last 2 years. Simultaneously, researchers investigated more effective treatments. Tasuku Honjo, a Japanese researcher, discovered the T-cell suppressor receptor PD-1 in 1992, paving the way for the development of negative immunoregulatory cancer therapies (43). Camrelizumab is one of the most representative drugs. Catilizumab was approved as a secondline drug for the systematic treatment of liver cancer by the National Medical Products Administration (NMPA) in 2020 based on the findings of various clinical trials (31). This adds a new treatment option to the treatment options for patients with advanced liver cancer. Clinical trials on immune checkpoint inhibitors are currently being conducted in large numbers, and the treatment of liver cancer has also come to the immune era (44). This may possibly be why Catilizumab has developed a reputation as a research hotspot. However, immune checkpoint inhibitors are likely to have limited activity as monotherapy in the majority of unselected HCC patients, which makes researchers consider the prospect of combined therapy (45). Anti-VEGF antibodies normalize tumor blood vessels, allowing T cells to invade tumors more effectively, while VEGF inhibitors can reprogram TME into immunostimulatory environments (30, 46). Antibodies against PD-1 and PDL-1 can increase $\mathrm{T}$ cell's ability to target tumors. These mechanisms provide a theoretical basis for the combination of targeted therapy and immunotherapy. The results of IMbrave150 demonstrate that Atezolizumab combined with bevacizumab is a reasonable treatment option for patients with advanced unresectable liver cancer (47). Subsequently, the FDA approved atezolizumab in combination with bevacizumab as a first-line therapeutic option for patients with advanced unresectable liver cancer. These findings aroused researchers' enthusiasm for the exploration of combined therapies, and novel combinations of various therapies have been proposed, including PD-1/PD-L1 immunotherapy in combination with CTLA-4 immune checkpoint inhibitors, transhepatic artery chemoembolization (TACE), or hepatic artery infusion chemotherapy (HAIC) in combination with immune checkpoint inhibitors or targeted therapy, and others $(48,49)$. Without a doubt, combination therapy is a current research hotspot.

Unfortunately, not all patients with HCC benefit from immunotherapy or combination therapy, which may be due to differences in their immunological environments (50). The TME is composed of immune cells, stromal cells, endothelial cells, inflammatory mediators, and extracellular matrix molecules, with immune cells and stromal cells being the two major nontumor components (51). Stromal and immune scores could be calculated by estimation of stromal and immune cells in malignant tumor tissues using expression data (ESTIMATE) (52). Immune scores are an excellent marker for predicting neoplastic outcomes and guiding clinicians in making treatment decisions (53). Therefore, it is critical to develop reasonable and accurate biomarkers for immunotherapy in combination with other therapies, to attain precision medical treatment.

Additionally, we used CiteSpace to identify burst keywords, which may serve as an essential indicator of research hotspots or research frontiers over time. As shown in Figure $\mathbf{9 B}$, the evolution of burst keywords over the past decade demonstrates the field's continued progress in HCC immunotherapy research. Based on this, we found that the keyword "landscape" has been confirmed as the strongest burst keyword in 2019. The term "landscape" refers primarily to the immunological and metabolic landscapes associated with cancer. As is well known, cancer immunotherapy is a relatively new and promising treatment modality that has emerged in recent years. While this treatment is capable of producing significant clinical effects, the tumor types were diverse and heterogeneity was unavoidable. Therefore, establishing a systematic and detailed tumor immunological landscape can aid in the development of tumor immunotherapy, the identification of effective targets and biomarkers (54), and the understanding of immune escape and drug resistance (55).

Bibliometric study is a method that depicts the evolution of scientific knowledge and its structural relationships, illustrating numerous implicit complicated relationships among clusters of knowledge (56). Thus, by comprehending these intricate knowledge relationships, researchers can understand the trend of knowledge in a specific field. Our bibliometric study elucidated that the discovery of additional immune targets, the elucidation of immune characteristics of the immune microenvironment in liver cancer, the exploration of biomarkers for predicting immunotherapy effect, and increased attempts to combine treatments may play a significant role in liver cancer immunotherapy in the following years.

\section{STRENGTHS AND LIMITATIONS}

For the first time, our study conducted a systematic analysis of HCC immunotherapy publications and their trend in an intuitive, objective, and accurate manner, which could serve as a comprehensive guide for clinicians and scholars working in this field. Simultaneously, we used a variety of bibliometrics software, to investigate research hotspots in multiple dimensions. Inevitably, there are some limitations in this study. First, the literature included in our study may not be exhaustive. On the one hand, our study investigated data from the WoSCC exclusively; data from other significant search engines such as PubMed, Embase, and Ovid were excluded. On the other hand, because the articles retrieved were limited to those published in English, some linguistic bias was introduced. Thus, the articles identified may not adequately reflect represent all HCC immunotherapy research. Second, due to their low citation rate, recently published high-quality articles may not receive the attention they deserve. This also demonstrates the 
importance of future research updates. Finally, while publications in the year 2021 were excluded due to insufficient data, this study includes the vast majority of papers published in the field of HCC immunotherapy between 2011 and 2020; new data may have few effects on the final results.

\section{CONCLUSION}

In general, advanced liver cancer treatment has entered a personalized precision treatment phase. Understanding the immune context of liver cancer by detecting the immune landscape of the disease, screening the population who may benefit from immunotherapy by calculating immune scores, and evaluating the efficacy of combined treatment strategies, particularly combinations of immune checkpoint inhibitors and other drugs, are urgent questions that need to be addressed urgently and also the future directions of immunotherapy research for liver cancer.

\section{REFERENCES}

1. Singal AG, Lampertico P, Nahon P. Epidemiology and Surveillance for Hepatocellular Carcinoma: New Trends. J Hepatol (2020) 72(2):250-61. doi: 10.1016/j.jhep.2019.08.025

2. Pascual S, Herrera I, Irurzun J. New Advances in Hepatocellular Carcinoma. World J Hepatol (2016) 8(9):421-38. doi: 10.4254/wjh.v8.i9.421

3. Mizukoshi E, Kaneko S. Immune Cell Therapy for Hepatocellular Carcinoma. J Hematol Oncol (2019) 12(1):52. doi: 10.1186/s13045-019-0742-5

4. Schachtschneider KM, Schwind RM, Darfour-Oduro KA, De AK, Rund LA, Singh K, et al. Transitional and Translational Porcine Model of Hepatocellular Carcinoma. Oncotarget (2017) 8(38):63620-34. doi: 10.18632/ oncotarget. 18872

5. El-Khoueiry AB, Sangro B, Yau T, Crocenzi TS, Kudo M, Hsu C, et al. Nivolumab in Patients With Advanced Hepatocellular Carcinoma (CheckMate 040): An Open-Label, Non-Comparative, Phase 1/2 Dose Escalation and Expansion Trial. Lancet (2017) 389(10088):2492-502. doi: 10.1016/s0140-6736(17)31046-2

6. Zhu AX, Finn RS, Edeline J, Cattan S, Ogasawara S, Palmer D, et al. Pembrolizumab in Patients With Advanced Hepatocellular Carcinoma Previously Treated With Sorafenib (KEYNOTE-224): A Non-Randomised, Open-Label Phase 2 Trial. Lancet Oncol (2018) 19(7):940-52. doi: 10.1016/ s1470-2045(18)30351-6

7. Rizzo A, Brandi G. Biochemical Predictors of Response to Immune Checkpoint Inhibitors in Unresectable Hepatocellular Carcinoma. Cancer Treat Res Commun (2021) 27:100328. doi: 10.1016/j.ctarc.2021.100328

8. Rizzo A, Dadduzio V, Ricci AD, Massari F, Di Federico A, Gadaleta-Caldarola G, et al. Lenvatinib Plus Pembrolizumab: The Next Frontier for the Treatment of Hepatocellular Carcinoma? Expert Opin Investig Drugs (2021) 30:1-8. doi: 10.1080/13543784.2021.1948532

9. Llovet JM, Kelley RK, Villanueva A, Singal AG, Pikarsky E, Roayaie S, et al. Hepatocellular Carcinoma. Nat Rev Dis Primers (2021) 7(1):6. doi: 10.1038/ s41572-020-00240-3

10. Rizzo A, Ricci AD, Brandi G. Immune-Based Combinations for Advanced Hepatocellular Carcinoma: Shaping the Direction of First-Line Therapy. Future Oncol (2021) 17(7):755-7. doi: 10.2217/fon-2020-0986

11. Inarrairaegui M, Melero I, Sangro B. Immunotherapy of Hepatocellular Carcinoma: Facts and Hopes. Clin Cancer Res (2018) 24(7):1518-24. doi: 10.1158/1078-0432.CCR-17-0289

12. Feng LF, Yan PJ, Chu XJ, Zhang N, Li JY, Li JW, et al. A Scientometric Study of the Top 100 Most-Cited Publications Based on Web-Of-Science Regarding Robotic Versus Laparoscopic Surgery. Asian J Surg (2021) 44(2):440-51. doi: 10.1016/j.asjsur.2020.10.026

\section{DATA AVAILABILITY STATEMENT}

The original contributions presented in the study are included in the article/Supplementary Material. Further inquiries can be directed to the corresponding authors.

\section{AUTHOR CONTRIBUTIONS}

$\mathrm{YH}, \mathrm{BL}$, JS, and HS designed this study. JS and HS performed the search and collected data. LK, XD, and JC re-checked data. JS and LK performed analysis. All authors contributed to the article and approved the submitted version.

\section{SUPPLEMENTARY MATERIAL}

The Supplementary Material for this article can be found online at: https://www.frontiersin.org/articles/10.3389/fimmu.2022. 815575/full\#supplementary-material

13. Agarwal A, Baskaran S, Panner Selvam MK, Barbarosie C, Master K. Unraveling the Footsteps of Proteomics in Male Reproductive Research: A Scientometric Approach. Antioxid Redox Signal (2020) 32(8):536-49. doi: 10.1089/ars.2019.7945

14. Synnestvedt MB, Chen C, Holmes JH. CiteSpace II: Visualization and Knowledge Discovery in Bibliographic Databases. AMIA Annu Symposium Proc AMIA Symposium (2005) 2005:724-8.

15. van Eck NJ, Waltman L. Citation-Based Clustering of Publications Using CitNetExplorer and VOSviewer. Scientometrics (2017) 111(2):1053-70. doi: 10.1007/s11192-017-2300-7

16. Guo Y, Hao Z, Zhao S, Gong J, Yang F. Artificial Intelligence in Health Care: Bibliometric Analysis. J Med Internet Res (2020) 22(7):e18228. doi: 10.2196/ 18228

17. Fang J, Pan L, Gu QX, Juengpanich S, Zheng JH, Tong CH, et al. Scientometric Analysis of mTOR Signaling Pathway in Liver Disease. Ann Transl Med (2020) 8(4):93. doi: 10.21037/atm.2019.12.110

18. Yang DW, Wang XP, Wang ZC, Yang ZH, Bian XF. A Scientometric Analysis on Hepatocellular Carcinoma Magnetic Resonance Imaging Research From 2008 to 2017. Quant Imaging Med Surg (2019) 9(3):465-76. doi: 10.21037/ qims.2019.02.10

19. Zhong D, Luo S, Zheng L, Zhang Y, Jin R. Epilepsy Occurrence and Circadian Rhythm: A Bibliometrics Study and Visualization Analysis via CiteSpace. Front Neurol (2020) 11:984. doi: 10.3389/fneur.2020.00984

20. Ke L, Lu C, Shen R, Lu T, Ma B, Hua Y. Knowledge Mapping of Drug-Induced Liver Injury: A Scientometric Investigation (2010-2019). Front Pharmacol (2020) 11:842. doi: 10.3389/fphar.2020.00842

21. Xie L, Chen Z, Wang H, Zheng C, Jiang J. Bibliometric and Visualized Analysis of Scientific Publications on Atlantoaxial Spine Surgery Based on Web of Science and VOSviewer. World Neurosurg (2020) 137:435-42.e4. doi: 10.1016/j.wneu.2020.01.171

22. Chen C, Hu Z, Liu S, Tseng H. Emerging Trends in Regenerative Medicine: A Scientometric Analysis in CiteSpace. Expert Opin Biol Ther (2012) 12(5):593608. doi: 10.1517/14712598.2012.674507

23. Jiang Q, Song X, Chen Z, Wang C, Luo H. Effects of Remifentanil on Hemodynamics, Liver Function and ICAM-1 Expression in Liver Cancer Patients Undergoing Surgery. Oncol Lett (2017) 14(1):872-6. doi: 10.3892/ ol.2017.6247

24. Bray F, Ferlay J, Soerjomataram I, Siegel RL, Torre LA, Jemal A. Global Cancer Statistics 2018: GLOBOCAN Estimates of Incidence and Mortality Worldwide for 36 Cancers in 185 Countries. CA Cancer J Clin (2018) 68(6):394-424. doi: $10.3322 /$ caac. 21492

25. European Association For The Study Of The L, European Organisation For R, Treatment Of C. EASL-EORTC Clinical Practice Guidelines: Management of 
Hepatocellular Carcinoma. J Hepatol (2012) 56(4):908-43. doi: 10.1016/ j.jhep.2011.12.001

26. Zucman-Rossi J, Villanueva A, Nault JC, Llovet JM. Genetic Landscape and Biomarkers of Hepatocellular Carcinoma. Gastroenterology (2015) 149 (5):1226-39.e4. doi: 10.1053/j.gastro.2015.05.061

27. Ruiz de Galarreta M, Bresnahan E, Molina-Sanchez P, Lindblad KE, Maier B, Sia D, et al. Beta-Catenin Activation Promotes Immune Escape and Resistance to Anti-PD-1 Therapy in Hepatocellular Carcinoma. Cancer Discov (2019) 9 (8):1124-41. doi: 10.1158/2159-8290.CD-19-0074

28. Forner A, Reig M, Bruix J. Hepatocellular Carcinoma. Lancet (2018) 391 (10127):1301-14. doi: 10.1016/s0140-6736(18)30010-2

29. Bruix J, Reig M, Sherman M. Evidence-Based Diagnosis, Staging, and Treatment of Patients With Hepatocellular Carcinoma. Gastroenterology (2016) 150(4):835-53. doi: 10.1053/j.gastro.2015.12.041

30. Gabrilovich DI, Chen HL, Girgis KR, Cunningham HT, Meny GM, Nadaf S, et al. Production of Vascular Endothelial Growth Factor by Human Tumors Inhibits the Functional Maturation of Dendritic Cells. Nat Med (1996) 2 (10):1096-103. doi: 10.1038/nm1096-1096

31. Chen Z, Lu X, Koral K. The Clinical Application of Camrelizumab on Advanced Hepatocellular Carcinoma. Expert Rev Gastroenterol Hepatol (2020) 14(11):1017-24. doi: 10.1080/17474124.2020.1807939

32. Small H. Co-Citation in the Scientific Literature: A New Measure of the Relationship Between Two Documents. J Am Soc Inf Sci (1973) 24(4):265-9. doi: 10.1002/asi.4630240406

33. Topalian SL, Hodi FS, Brahmer JR, Gettinger SN, Smith DC, McDermott DF, et al. Safety, Activity, and Immune Correlates of Anti-PD-1 Antibody in Cancer. N Engl J Med (2012) 366(26):2443-54. doi: 10.1056/NEJMoa1200690

34. Sangro B, Gomez-Martin C, de la Mata M, Inarrairaegui M, Garralda E, Barrera P, et al. A Clinical Trial of CTLA-4 Blockade With Tremelimumab in Patients With Hepatocellular Carcinoma and Chronic Hepatitis C. J Hepatol (2013) 59(1):81-8. doi: 10.1016/j.jhep.2013.02.022

35. Bruix J, Qin S, Merle P, Granito A, Huang Y-H, Bodoky G, et al. Regorafenib for Patients With Hepatocellular Carcinoma Who Progressed on Sorafenib Treatment (RESORCE): A Randomised, Double-Blind, Placebo-Controlled, Phase 3 Trial. Lancet (2017) 389(10064):56-66. doi: 10.1016/s0140-6736(16) 32453-9

36. Kudo M, Finn RS, Qin S, Han K-H, Ikeda K, Piscaglia F, et al. Lenvatinib Versus Sorafenib in First-Line Treatment of Patients With Unresectable Hepatocellular Carcinoma: A Randomised Phase 3 Non-Inferiority Trial. Lancet (2018) 391(10126):1163-73. doi: 10.1016/s0140-6736(18)30207-1

37. Ferlay J, Soerjomataram I, Dikshit R, Eser S, Mathers C, Rebelo M, et al. Cancer Incidence and Mortality Worldwide: Sources, Methods and Major Patterns in GLOBOCAN 2012. Int J Cancer (2015) 136(5):E359-86. doi: $10.1002 /$ ijc. 29210

38. Pardoll DM. The Blockade of Immune Checkpoints in Cancer Immunotherapy. Nat Rev Cancer (2012) 12(4):252-64. doi: 10.1038/nrc3239

39. Prieto J, Melero I, Sangro B. Immunological Landscape and Immunotherapy of Hepatocellular Carcinoma. Nat Rev Gastroenterol Hepatol (2015) 12 (12):681-700. doi: 10.1038/nrgastro.2015.173

40. Regorafenib Approved for Liver Cancer. Cancer Discov (2017) 7(7):660. doi: 10.1158/2159-8290.Cd-nb2017-070

41. Tang W, Chen Z, Zhang W, Cheng Y, Zhang B, Wu F, et al. The Mechanisms of Sorafenib Resistance in Hepatocellular Carcinoma: Theoretical Basis and Therapeutic Aspects. Signal Transduct Target Ther (2020) 5(1):87. doi: 10.1038/s41392-020-0187-x

42. Facciorusso A, Abd El Aziz MA, Sacco R. Efficacy of Regorafenib in Hepatocellular Carcinoma Patients: A Systematic Review and MetaAnalysis. Cancers (2019) 12(1). doi: 10.3390/cancers12010036

43. Granier C, Karaki S, Roussel H, Badoual C, Tran T, Anson M, et al. Cancer Immunotherapy: Rational and Recent Breakthroughs. La Rev Med Interne (2016) 37(10):694-700. doi: 10.1016/j.revmed.2016.05.023. Immunothérapie des cancers: rationnel et avancées récentes.
44. Xu F, Jin T, Zhu Y, Dai C. Immune Checkpoint Therapy in Liver Cancer. J Exp Clin Cancer Res: CR (2018) 37(1):110. doi: 10.1186/s13046-018-0777-4

45. Uson Junior PLS, Liu AJ, Sonbol MB, Borad MJ, Bekaii-Saab TS. Immunotherapy and Chimeric Antigen Receptor T-Cell Therapy in Hepatocellular Carcinoma. Chin Clin Oncol (2021) 10(1):11. doi: 10.21037/cco-20-231

46. Hegde PS, Wallin JJ, Mancao C. Predictive Markers of Anti-VEGF and Emerging Role of Angiogenesis Inhibitors as Immunotherapeutics. Semin Cancer Biol (2018) 52(Pt 2):117-24. doi: 10.1016/j.semcancer.2017.12.002

47. Qin S, Ren Z, Feng YH, Yau T, Wang B, Zhao H, et al. Atezolizumab Plus Bevacizumab Versus Sorafenib in the Chinese Subpopulation With Unresectable Hepatocellular Carcinoma: Phase 3 Randomized, Open-Label IMbrave150 Study. Liver Cancer (2021) 10(4):296-308. doi: 10.1159/ 000513486

48. Zhou H, Song T. Conversion Therapy and Maintenance Therapy for Primary Hepatocellular Carcinoma. Biosci Trends (2021) 15(3):155-60. doi: 10.5582/ bst.2021.01091

49. El Dika I, Khalil DN, Abou-Alfa GK. Immune Checkpoint Inhibitors for Hepatocellular Carcinoma. Cancer (2019) 125(19):3312-9. doi: 10.1002/ cncr.32076

50. Yang JD, Hainaut P, Gores GJ, Amadou A, Plymoth A, Roberts LR. A Global View of Hepatocellular Carcinoma: Trends, Risk, Prevention and Management. Nat Rev Gastroenterol Hepatol (2019) 16(10):589-604. doi: 10.1038/s41575-019-0186-y

51. Hanahan D, Coussens LM. Accessories to the Crime: Functions of Cells Recruited to the Tumor Microenvironment. Cancer Cell (2012) 21(3):309-22. doi: 10.1016/j.ccr.2012.02.022

52. Yoshihara K, Shahmoradgoli M, Martínez E, Vegesna R, Kim H, TorresGarcia W, et al. Inferring Tumour Purity and Stromal and Immune Cell Admixture From Expression Data. Nat Commun (2013) 4:2612. doi: 10.1038/ ncomms 3612

53. Bruni D, Angell HK, Galon J. The Immune Contexture and Immunoscore in Cancer Prognosis and Therapeutic Efficacy. Nat Rev Cancer (2020) 20 (11):662-80. doi: 10.1038/s41568-020-0285-7

54. Sun Y, Wu L, Zhong Y, Zhou K, Hou Y, Wang Z, et al. Single-Cell Landscape of the Ecosystem in Early-Relapse Hepatocellular Carcinoma. Cell (2021) 184 (2):404-21.e16. doi: 10.1016/j.cell.2020.11.041

55. Qiu Z, Li H, Zhang Z, Zhu Z, He S, Wang X, et al. A Pharmacogenomic Landscape in Human Liver Cancers. Cancer Cell (2019) 36(2):179-93.e11. doi: 10.1016/j.ccell.2019.07.001

56. Zou X, Yue WL, Vu HL. Visualization and Analysis of Mapping Knowledge Domain of Road Safety Studies. Accid Anal Prev (2018) 118:131-45. doi: 10.1016/j.aap.2018.06.010

Conflict of Interest: The authors declare that the research was conducted in the absence of any commercial or financial relationships that could be construed as a potential conflict of interest.

The reviewer GW declared a shared parent affiliation with the author(s) to the handling editor at the time of the review.

Publisher's Note: All claims expressed in this article are solely those of the authors and do not necessarily represent those of their affiliated organizations, or those of the publisher, the editors and the reviewers. Any product that may be evaluated in this article, or claim that may be made by its manufacturer, is not guaranteed or endorsed by the publisher.

Copyright $(2022$ Shen, Shen, Ke, Chen, Dang, Liu and Hua. This is an open-access article distributed under the terms of the Creative Commons Attribution License (CC BY). The use, distribution or reproduction in other forums is permitted, provided the original author(s) and the copyright owner(s) are credited and that the original publication in this journal is cited, in accordance with accepted academic practice. No use, distribution or reproduction is permitted which does not comply with these terms. 